\title{
An observer-based control of linear systems with uncertain parameters
}

\author{
MOSTAFA RACHIK and MUSTAPHA LHOUS
}

In this paper, the observer-based control for a class of uncertain linear systems is considered. Exponential stabilizability for the system is studied and reduced-order observer is discussed. Numerical examples are given to illustrate obtained results.

Key words: observer-based control, stability, uncertain linear systems.

\section{Introduction}

The state of a system are not always measurable in many control systems and application. The design of a system that produces an approximation to the state vector, this system is called an observer has as its inputs the inputs and available output of the system whose state is to be approximated and has a state vector that is linearly related to the desired approximation. The observer-based control will be used to reconstruct the state of a dynamic system. The observer was developed firstly for deterministic continuoustime linear time-invariant system and has been extended by several researchers to timevaring, discrete and stochastic systems [1], [2], [7], [12], [13], [14], [15], [16], [17], [18] and [19]. The simplicity of its design and its resolution of the difficulty imposed by missing measurements make the observer an attractive general design component.

It is well know that in many practical control systems, the system almost presents some uncertainties and perturbations, may be due to additive unknown internal or external noise, environmental influence, nonlinearities, data errors, uncertain or slowly varying parameters, etc.

Recently, many work has been devoted to design the observer or observer-based control of uncertain system [3], [8], [10], [11] and [12]. In [2] and [7] the lyapunov stability theory is used to design the state observer for linear time varying or nonlinear

M. Rachik is with Laboratory of Analysis Modeling and Simulation, Department of Mathematics and Computer Science, Faculty of Sciences Ben M'sik, Hassan II University of Casablanca, B.P 7955 Sidi Othman Casablanca, Morocco. E-mail: m_rachik@yahoo.fr. M. Lhous is with Laboratory of Modeling, Analysis, Control and Statistics, Department of Mathematics and Computer Science, Faculty of Sciences Ain Chock, Hassan II University of Casablanca, B.P 5366 Maarif Casablanca, Morocco. E-mail: mlhous17@gmail.com

Received 06.06.2016. 
systems. Linear matrix inequality (LMI) approach have been used for output feedback control [8], [10], [11] and [12].

In this paper and inspired by the work of [4], we well adopt the useful methodologies used in [4] to the design of the observer-based control for the class of following uncertain systems.

$$
\left\{\begin{aligned}
\dot{x}(t)= & (A+\Delta A) x(t)+B u(t) \\
x(0) & x_{0}
\end{aligned}\right.
$$

The control and observer gains could be satisfied some assumptions.

The paper is organized as follows. In section 2, we provide the problem formulation and main results concerning the observer-based control for systems with state perturbations. A numerical example is given in section 3 to illustrate the proposed results. Finally, we discus a reduced-order observer in section 4.

Notation. Throughout this paper, we use the following notations

- $\mathfrak{R}^{n} n$-dimensional real space.

- $\mathcal{M}_{n, m}(\Re)$ set of all real $n$ by $m$ matrices.

- $\lambda_{i}(A)$ eigenvalue of real matrix $A$.

- $S p(A)$ set of all eigenvalues of real matrix $A$.

- $\|A\|$ the infinity-norm of matrix $A$.

- I identity matrix with appropriate dimension.

- 0 zero matrix with appropriate dimension.

\section{Problem formulation and Main results}

Consider a continuous uncertain linear system described by

$$
\left\{\begin{aligned}
\dot{x}(t)= & (A+\Delta A) x(t)+B u(t) \\
x(0) & x_{0}
\end{aligned}\right.
$$

where $x(t) \in \mathfrak{R}^{n}$ is the state vector, $u(t) \in \mathfrak{R}^{m}$ is the control input vector. $A \in \mathfrak{M}_{n}(\mathfrak{R})$, $B \in \mathcal{M}_{n, m}(\Re)$ are constant matrices and $\Delta A \in \mathcal{M}_{n}(\mathfrak{R})$ is a perturbed matrix. The uncertain parameter $\Delta A$ will represent the impossibility for exact mathematical model of a dynamic system due to the system complexity.

The output equation is given by

$$
y(t)=C x(t)
$$


where $C \in \mathcal{M}_{p, n}(\Re)$.

A suitable dynamic observer-based control of the system (1)-(2) is given by

$$
\left\{\begin{array}{l}
\dot{\hat{x}}(t)=\hat{A} \hat{x}(t)+\hat{B} u(t)+L(y(t)-\hat{y}(t)) \\
\hat{y}(t)=C \hat{x}(t) \\
u(t)=-K \hat{x}(t)
\end{array}\right.
$$

where $\hat{x}(t) \in \mathfrak{R}^{n}$ is the estimation of $x(t), \hat{y}(t) \in \mathfrak{R}^{p}$ is the observer output, $K \in \mathcal{M}_{m, n}(\mathfrak{R})$ is the control gain, $L \in \mathcal{M}_{n, p}(\mathfrak{R})$ is the observer gain, $\hat{B} \in \mathcal{M}_{n, m}(\mathfrak{R})$.

By (3), (1) with (2) can be rewritten as

$$
\dot{x}(t)=(A-B K+\Delta A) x(t)+B K e(t)
$$

where $e(t)=x(t)-\hat{x}(t)$ is the estimated error.

$$
\dot{e}(t)=[A-\hat{A}-(B-\hat{B}) K+\Delta A]) x(t)+[\hat{A}-L C+(B-\hat{B}) K] e(t)
$$

Define $z(t)=\left[\begin{array}{l}x(t) \\ e(t)\end{array}\right]$, then we have

$$
\begin{aligned}
\dot{z}(t) & =\left[\begin{array}{l}
\hat{x(t)} \\
e(t)
\end{array}\right] \\
& =\left[\begin{array}{cc}
A-B K+\Delta A & B K \\
A-\hat{A}-(B-\hat{B}) K+\Delta A & \hat{A}-L C+(B-\hat{B}) K
\end{array}\right] z(t) \\
& =\left[\begin{array}{cc}
A-B K & 0 \\
A+\Delta A-\hat{A}-(B-\hat{B}) K & \hat{A}-L C
\end{array}\right] z(t)+\left[\begin{array}{cc}
\Delta A & B K \\
0 & (B-\hat{B}) K
\end{array}\right] z(t)
\end{aligned}
$$

thus, we can write

$$
\dot{z}(t)=(\bar{A}+\bar{B}) z(t)
$$

with

$$
\bar{A}=\left[\begin{array}{cc}
A-B K & 0 \\
A+\Delta A-\hat{A}-(B-\hat{B}) K & \hat{A}-L C
\end{array}\right] \text { and } \bar{B}=\left[\begin{array}{cc}
\Delta A & B K \\
0 & (B-\hat{B}) K
\end{array}\right]
$$

Assertion 1: Suppose that

- The pair $(A, B)$ is stabilizable $(A-B K$ is Hurwitz).

- The pair $(\hat{A}, C)$ is detectable $(\hat{A}-L C$ is Hurwitz). 
Proposition 1 If Assertion 1 holds then the block triangular matrix $\bar{A}$ is also a stability matrix with stability abscissa $(-\rho)$ subject to the constraint

$$
\max \left\{\operatorname{Re}\left(\lambda_{i}(A-B K)\right), \operatorname{Re}\left(\lambda_{i}(\hat{A}-L C)\right)\right\} \leqslant-\rho<0
$$

where the first inequality is non strict if there is some multiple eigenvalue of $\bar{A}$.

Proof Stability abscissa of $\bar{A}$ is the largest of the real parts of all the characteristic roots

$$
\begin{aligned}
-\rho= & \max \{\operatorname{Re}(s) \mid \operatorname{Re}(\bar{A}-s I)=0\}=\max _{i}(\bar{A}) \\
\operatorname{det}(\bar{A}-\lambda I) & =\operatorname{det}\left[\begin{array}{cc}
A-B K-\lambda I & 0 \\
A+\Delta A-\hat{A}-(B-\hat{B}) K & \hat{A}-L C-\lambda I
\end{array}\right] \\
& =\operatorname{det}(A-B K-\lambda I) \operatorname{det}(\hat{A}-L C-\lambda I)
\end{aligned}
$$

then

$$
\operatorname{sp}(\bar{A})=\operatorname{sp}(A-B K) \cup \operatorname{sp}(\hat{A}-L C)
$$

and with assertion 1 , we have

$$
\max \left\{\operatorname{Re}\left(\lambda_{i}(A-B K)\right), \operatorname{Re}\left(\lambda_{i}(\hat{A}-L C)\right)\right\}<0 .
$$

Consequently, the matrix $\bar{A}$ is stable.

Remark 1 (Pole Placement) If the pair $(A, B)$ is controllable, then there exists a feedback gain matrix $\mathrm{K}$ that arbitrarily assigns the system poles to any set $\left\{\lambda_{i}, i=1, \ldots, n\right\}$. Furthermore, if the pair (A, B) is stabilizable, then the controllable modes can all be arbitrarily assigned.

Proposition 2 The matrix $(\bar{A}+\bar{B})$ is stable if $\bar{A}$ is a stability matrix and $\|\bar{B}\| \leqslant \rho$ where $(-\rho)$ is the stability abscissa of the matrix $\bar{A}$.

Proof The solution of system (4) is given by

$$
z(t)=e^{\bar{A} t} z(0)+\int_{0}^{t} e^{\bar{A}(t-s)} \bar{B} z(s) d s .
$$

Since $\bar{A}$ is stable then $\left\|e^{\bar{A} t}\right\| \leqslant k_{0} e^{-\rho t}$, with some real constant $k_{0} \geqslant 1$ (norm-dependent). If we consider $v(t)=e^{-\rho t}\|z(t)\|$, then we have

$$
\begin{aligned}
\|z(t)\| & =\left\|e^{\bar{A} t} z(0)+\int_{0}^{t} e^{\bar{A}(t-s)} \bar{B} z(s) d s\right\| \\
& \leqslant\left\|e^{\bar{A} t}\right\|\|z(0)\|+\int_{0}^{t}\left\|e^{\bar{A}(t-s)}\right\|\|\bar{B}\|\|z(s)\| d s \\
& \leqslant k_{0} e^{-\rho t}\|z(0)\|+\int_{0}^{t} k_{0} e^{-\rho(t-s)}\|\bar{B}\|\|z(s)\| d s
\end{aligned}
$$


thus

$$
v(t) \leqslant k_{0}\left[\|z(0)\|+\int_{0}^{t}\|\bar{B}\| v(s) d s\right] \text { all } t \geqslant 0 .
$$

Using Gronwall's Lemma [5] and [6] in (6)

$$
\begin{aligned}
\|z(t)\| \leqslant e^{-\rho t} v(t) & \leqslant k_{0}\|z(0)\| \exp \left(-\rho t+\int_{0}^{t}\|\bar{B}\| d s\right) \\
& \leqslant k_{0}\|z(0)\| e^{-(\rho-\|\bar{B}\|) t}
\end{aligned}
$$

for all $t \geqslant 0$. Then the matrix $(\bar{A}+\bar{B})$ is stable if $\|\bar{B}\| \leqslant \rho$.

From assertion 1 and proposition 2 the following follows

Proposition 3 System (1)-(2) is exponentially stabilizable by (3) if the pair $(A, B)$ and $(\hat{A}, C)$ are respectively stabilizable and detectable and $\|\bar{B}\| \leqslant \rho$ where $(-\rho)$ is the stability abscissa of the matrix $\bar{A}$.

\section{Simulation example}

Let us consider the two compartment model

$$
\begin{aligned}
& \dot{x}_{1}=-\left(k_{01}+k_{21}\right) x_{1}+k_{12} x_{2}+u \\
& \dot{x}_{2}=k_{21} x_{1}+-\left(k_{02}+k_{12}\right) x_{2}
\end{aligned}
$$

where the output equation is

$$
y=x_{1}
$$

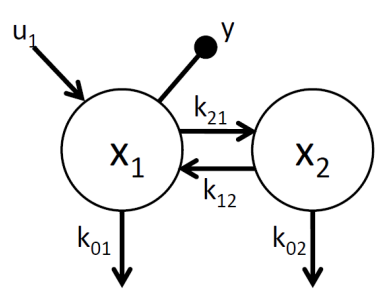

Figure 1: Linear 2-compartment model. 
The two parameters $k_{12}$ and $k_{21}$ are assumed to be uncertain and given by $\hat{k}_{12}=$ $k_{12}+\varepsilon_{1}$, and $\hat{k}_{21}=k_{21}+\varepsilon_{2}$. The overall system can be rewritten as

$$
\begin{aligned}
\dot{x}_{1} & =-\left(k_{01}+\hat{k}_{21}\right) x_{1}+\hat{k}_{12} x_{2}+u \\
\dot{x}_{2} & =\hat{k}_{21} x_{1}+-\left(k_{02}+\hat{k}_{12}\right) x_{2} \\
y & =x_{1}
\end{aligned}
$$

Denoting $x(t)=\left[\begin{array}{l}x_{1} \\ x_{2}\end{array}\right]$, the system can be rewritten as

$$
\dot{x}(t)=(A+\Delta) x(t)+B u(t)
$$

with

$$
A=\left(\begin{array}{cc}
-k_{01}-k_{21} & k_{12} \\
k_{21} & -k_{02}-k_{12}
\end{array}\right), \Delta A=\left(\begin{array}{cc}
\varepsilon_{2} & \varepsilon_{1} \\
\varepsilon_{2} & \varepsilon_{1}
\end{array}\right) \text { and } B=\left[\begin{array}{l}
1 \\
0
\end{array}\right] .
$$

Let us consider

$$
k_{01}=2, k_{02}=1.4, k_{12}=1, k_{21}=-2.3, \varepsilon_{1}=0.3 \text { and } \varepsilon_{2}=0.4
$$

for numerical application. Moreover, an estimation of the state and the control, observer gains ara given by

$$
\hat{A}=\left[\begin{array}{cc}
0.60 & 1.08 \\
-1.8 & -2.5
\end{array}\right], K=\left[\begin{array}{ll}
0.02 & 0.07
\end{array}\right] \text { and } L=\left[\begin{array}{c}
1 \\
0.8
\end{array}\right]
$$

System (9) with (10) would be exponentially stabilized by (3) with (11) and the stability abscissa of the matrix $\bar{A}$ is $-\rho=1.06$ and $\|\bar{B}\|=0.579$. With $x(0)=\left[\begin{array}{c}-1 \\ 2\end{array}\right]$ and $\hat{x}(0)=\left[\begin{array}{l}1 \\ 1\end{array}\right]$ some state trajectories for uncontrolled approximation of the system by an observer-based control and the estimated error are depicted in Figs 2 and 3 respectively.

\section{Reduced dimension observer}

Consider the uncertain linear system

$$
\begin{cases}\dot{x}(t)= & (A+\Delta A) x(t)+B u(t) \\ x(0) & x_{0}\end{cases}
$$



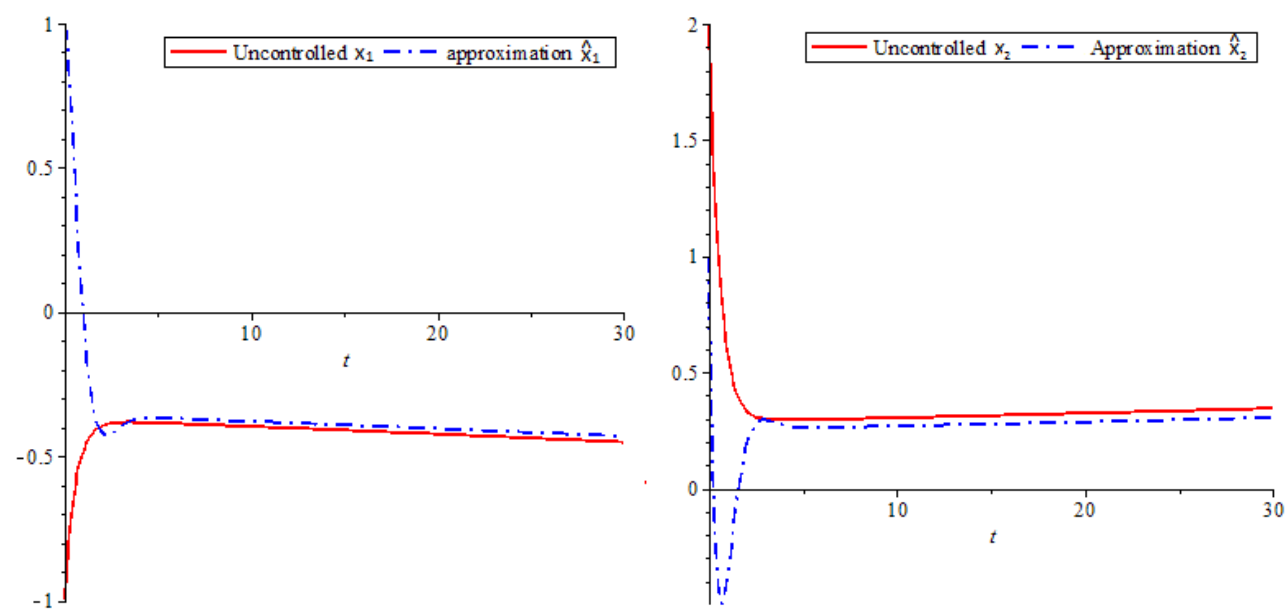

Figure 2: Trajectories of the uncontrolled and the approximation of system.
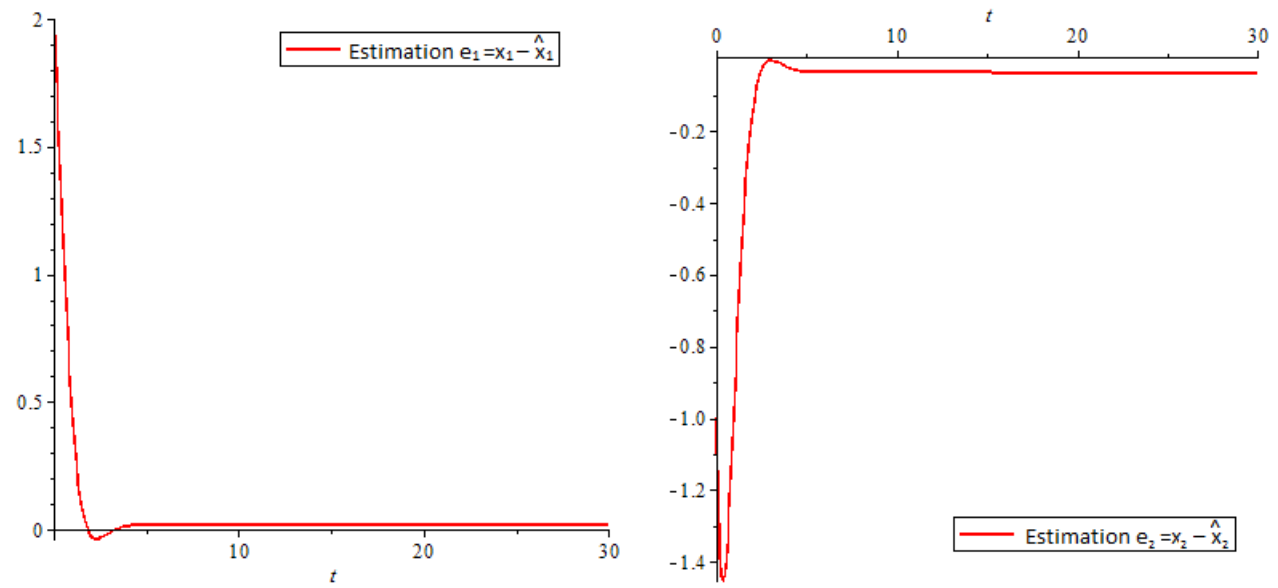

Figure 3: Trajectories of the estimated error.

where $x(t) \in \mathfrak{R}^{n}$ is the state vector, $u(t) \in \mathfrak{R}^{m}$ is the control input vector. $A \in \mathcal{M}_{n}(\mathfrak{R})$, $B \in \mathcal{M}_{n, m}(\mathfrak{R})$ are constant matrices and $\Delta A \in \mathcal{M}_{n}(\mathfrak{R})$ is a perturbed matrix.

The output equation is given by

$$
y(t)=C x(t)
$$

where $C \in \mathcal{M}_{p, n}(\Re)$. 
Let $\Gamma \in \mathcal{M}_{q, n}(\mathfrak{R})$, we search the estimation $\hat{x} \in \mathfrak{R}^{q}$ of $\Gamma x$. A suitable dynamic observer-based control of the system (12)-(13) is given by

$$
\left\{\begin{array}{l}
\dot{\hat{x}}(t)=\hat{A} \hat{x}(t)+\hat{B} u(t)+L(y(t)-\hat{y}(t)) \\
\hat{y}(t)=C_{1} \hat{x}(t) \\
u(t)=-K \hat{x}(t)
\end{array}\right.
$$

where $\hat{x}(t) \in \mathfrak{R}^{q}$ is the estimation of $\Gamma x(t), \hat{y}(t) \in \mathfrak{R}^{p}$ is the observer output, $K \in \mathcal{M}_{m, q}(\Re)$ is the control gain, $L \in \mathcal{M}_{q, p}(\Re)$ is the observer gain, $\hat{B} \in \mathcal{M}_{q, m}(\Re)$ and $C_{1} \in \mathcal{M}_{p, q}(\Re)$ with $C=\left[C_{1} \mid C_{2}\right] . e(t)=\Gamma x(t)-\hat{x}(t)$ is the estimated error of system. By (12) and (13) with (14) we obtain

$$
\begin{aligned}
& \frac{d}{d t}\left[\begin{array}{l}
x(t) \\
e(t)
\end{array}\right]=\left[\begin{array}{cc}
A-B K \Gamma & 0 \\
D & \hat{A}-L C_{1}
\end{array}\right] z(t)+ \\
& {\left[\begin{array}{cc}
\Delta A & B K \\
0 & \Gamma B K-\hat{B} K
\end{array}\right] z(t)}
\end{aligned}
$$

where

$$
D=\Gamma A-\hat{A} \Gamma+\Gamma \Delta A+L C_{1} \Gamma-L C-\Gamma B K \Gamma+\hat{B} K \Gamma .
$$

Then, the equation can be written as

$$
\frac{d}{d t}\left[\begin{array}{l}
x(t) \\
e(t)
\end{array}\right](t)=(\bar{A}+\bar{B}) z(t)
$$

with

$$
\bar{A}=\left[\begin{array}{cc}
A-B K \Gamma & 0 \\
D & \hat{A}-L C_{1}
\end{array}\right]
$$

and

$$
\bar{B}=\left[\begin{array}{cc}
\Delta A & B K \\
0 & \Gamma B K-\hat{B} K
\end{array}\right] .
$$

We apply the result established in proposition 2 and 3 to give sufficient conditions which make the system (12)-(13) exponentially stabilizable by (14).

Proposition 4 System (12)-(13) is exponentially stabilizable by (14) if the matrices A$B K \Gamma$ and $\hat{A}-L C_{1}$ are stable and $\|\bar{B}\| \leqslant \rho$ where $(-\rho)$ is the stability abscissa of the matrix $\bar{A}$.

Example Let us consider the example of a simple gene expression process described by the following model [9]:

$$
\left[\begin{array}{c}
\dot{x}_{r}(t) \\
\dot{x}_{p}(t)
\end{array}\right]=\left[\begin{array}{cc}
-\gamma_{r} & 0 \\
k_{p} & -\gamma_{p}
\end{array}\right]\left[\begin{array}{c}
x_{r}(t) \\
x_{p}(t)
\end{array}\right]+\left[\begin{array}{l}
1 \\
0
\end{array}\right] u(t)
$$


where $x_{r} \geqslant 0$ is the mean number of mRNA in the cell, $x_{p} \geqslant 0$ is the mean number of protein of interest in the cell. Above $u(t) \geqslant 0$ is the transcription rate of DNA into mRNA, $\gamma_{r}>0$ is the degradation rate of mRNA, $k_{p}>0$ is the translation rate of mRNA into protein and $\gamma_{p}>0$ is the degradation rate of the protein. The parameters are assumed to be uncertain and given by $\gamma_{r}=\gamma_{r}^{0}+\varepsilon_{1} \gamma_{r}^{1}, k_{p}=k_{p}^{0}+\varepsilon_{2} k_{p}^{1}$ and $\gamma_{p}=\gamma_{p}^{0}+\varepsilon_{3} \gamma_{p}^{1}$, where $\varepsilon_{i} \in[-1,1], i=1,2,3$.

Let us consider $\gamma_{r}^{0}=1, k_{p}^{0}=2$ and $\gamma_{p}^{0}=1$ for numerical application. We also assume that the parameters are known up to a percentage $N \in[0,1)$ of their nominal value, hence $\gamma_{r}^{1}=N \gamma_{r}^{0}, \gamma_{p}^{1}=N \gamma_{p}^{0}$ and $k_{p}^{1}=N k_{p}^{0}$ with $N=\frac{1}{2}, \varepsilon_{1}=-0.5, \varepsilon_{2}=0.8$ and $\varepsilon_{1}=0.4$. Moreover, for

$$
C=\left[\begin{array}{ll}
1 & 0 \\
0 & 1
\end{array}\right], \Gamma=\left[\begin{array}{ll}
1 & 1
\end{array}\right] \text { and } \hat{A}=-0.3,
$$

the control and observer gains are given by

$$
K=0.005 \text { and } L=\left[\begin{array}{l}
1 \\
0
\end{array}\right]
$$

System (16) would be exponentially stabilized by (14) with (17) and the stability abscissa of the matrix $\bar{A}$ is $-\rho=1.002$ and $\|\bar{B}\|=1$.

With $x(0)=\left[\begin{array}{c}1 \\ -1\end{array}\right]$ and $\hat{x}(0)=1$ some state trajectories for uncontrolled approximation of the system by an observer-based control and the estimated error are depicted in Figs 4 and 5 respectively.

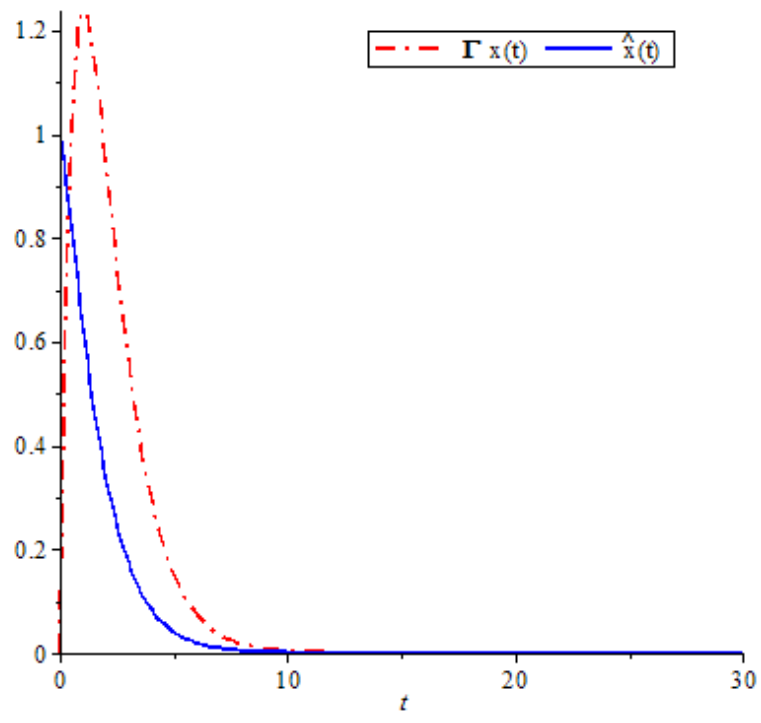

Figure 4: Trajectories of the uncontrolled and the approximation of system. 


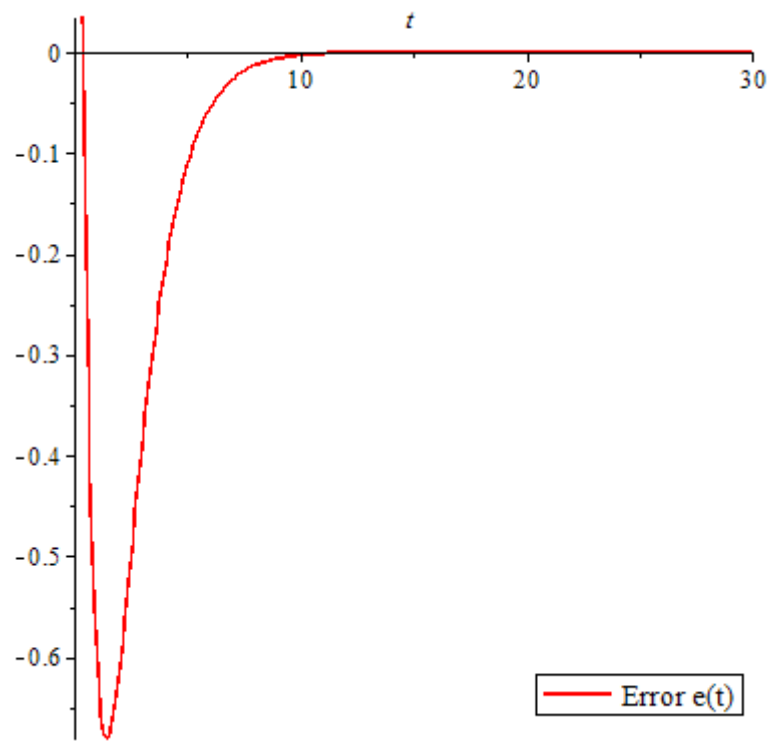

Figure 5: Trajectories of the estimated error.

\section{Conclusion}

In this paper, we have considered the observer-based control problem for a class of uncertain linear system. We give somme condition to quarantee that the feedbackcontrolled system is exponentially stabilizable by linear observer-based control. A numerical example has been given to demonstrate the use of the obtained results.

\section{References}

[1] M. AOKI and J.R. HUdDLE: Estimation of state vector of a linear stochastic system with a constrained estimator. IEEE Trans. on Automatic control, (Short papers), 12 (1967), 432-433.

[2] K.K. BUSAWON and M. SAIF: A state observer for nonlinear systems. IEEE Trans. on Automatic Control, 44 (1999), 2098-2103.

[3] M. ChEn and W-H. ChEn: Disturbance-observer-based robust control for time delay uncertain systems. Int. J. of Control, Automation and Systems, 8(2), (2010), 445-453. 
[4] M. De la Sen, S. Alonso-Quesada, A. Ibeas and R. Nistal: An observerbased vaccination law for a SEIR epidemic model. Int. J. of Computer Theory and Engineering, 4(3), (2012), 379-385.

[5] M. DE LA SEN: On impulsive time-varying systems with unbounded time-varying point delays: Stability and compacteness of the relevant operators mapping the input space into the state and output spaces. Rocky Mountain J. of Mathematics, 37 (2007), 79-129.

[6] M. DE LA SEN: The use of Gronwall's lemma for robust compensation of timevarying linear systems. Int. J. of Systems Science, 22(5), (1991), 885-903.

[7] D.W. GU and F.W. Poon: A robust state observer scheme. IEEE Trans. on Automatic Control, 46 (2001), 1958-1963.

[8] S. HUI and S.H. ZAK: Observer design for systems with unknown inputs. Int. J. of Applied Mathematics and Computer Science, 15(4), (2005), 431-446.

[9] M. Khammash: Modeling and analysis of stochastic biochemical networks. In Control Theory and Systems Biology, P.A. Iglesias and B.P. Ingalls (Eds.), MIT Press, (2010), 29-44.

[10] H. Kheloufi, A. Zemouche, F. Bedouhene and M. Boutayeb: A new observer-based stabilization method for linear systems with uncertain parameters. European Control Conf., Zurich, Switzerland, (2013), 1120-1125.

[11] C.H. LIEN: An efficient method to design robust observer-based control of uncertain linear systems. Applied Mathematics and Computation, 158 (2004), 29-44.

[12] C.H. LIEN: Robust observer-based control of systems with state perturbations via LMI approach. IEEE Trans. on Automatic control, 4(8), (2004), 1365-1370.

[13] D. Luenberger: Observing the state of a linear system. IEEE Trans. on Automatic Control, 8 (1964), 74-80.

[14] D. Luenberger: An introduction to observers. IEEE Trans. on Automatic Control, 16(6), (1971), 596-602.

[15] M. RachiK, S. SAadi, Y. Rahhou and O. El Kahlaoui: Observer design and admissible disturbances: A discrete disturbed system. Applied Mathematical Sciences, 1(33), (2007), 1631-1650.

[16] O. El Kahlaoui, S. SaAdi, Y. Rahhou, A. Bourass and M. RachiK: On the improvement of the performances of an observer: A discrete linear system. Applied Mathematical Sciences, 1(6), (2007), 241-266. 
[17] M. Rachik, H. LaARABi, O. El Kahlaoui and S. SAadi: Observer for perturbed linear continuous systems. Applied Mathematical Sciences, 4(14), (2010), 681-689.

[18] H. LaArabi, M. RachiK, N. Yousfi, J. BouYaghroumni and E. LabriJi: Observer for perturbed linear discrete systems. Int. Mathematical Forum, 5(41), (2010), 2023-2035.

[19] M.V. Thuan, V.N. Phat and H. Trinh: Observer-based controller design of time-delay systems with an interval time-varying delay. Int. J. of Applied Mathematics and Computer Science, 22(4), (2012), 921-927. 